\title{
ADDITIONAL PROPERTIES FOR WEAKLY Po AND RELATED PROPERTIES WITH AN APPLICATION
}

\section{CHARLES DORSETT}

Department of Mathematics

Texas A\&M University-Commerce

Texas 75429

USA

e-mail: charles.dorsett@tamuc.edu

\begin{abstract}
Within this paper, additional properties for weakly $P o$ and related properties are given and the results are applied to give an internal, well-defined topological properties $W P$ for which $W P=$ weaker $P o$ for each $P o$ that exists.
\end{abstract}

\section{Introduction and Preliminaries}

$T_{0}$-identification spaces were introduced by Stone in 1936 [12].

Definition 1.1. Let $(X, T)$ be a space, let $R$ be the equivalence relation on $X$ defined by $x R y$ iff $C l(\{x\})=C l(\{y\})$, let $X_{0}$ be the set of $R$ equivalence classes of $X$, let $N: X \rightarrow X_{0}$ be the natural map, and let $Q(X, T)$ be the decomposition topology on $X_{0}$ determined by $(X, T)$ and the map $N$. Then $\left(X_{0}, Q(X, Y)\right)$ is the $T_{0}$-identification space of $(X, T)$. 
Within the 1936 paper [12], $T_{0}$-identification spaces were used to further characterize metrizable spaces.

Theorem 1.1. A space $(X, T)$ is pseudometrizable iff $\left(X_{0}, Q(X, Q\right.$ $(X, T)))$, is metrizable. In the 1975 paper [11], $T_{0}$-identification spaces were used to further characterize Hausdorff spaces.

Theorem 1.2. A space $(X, T)$ is weakly Hausdorff iff $\left(X_{0}, Q(X, T)\right)$ is Hausdorff [9].

Within the 2015 paper [1], the metrizable and Hausdorff properties were generalized to weakly $P o$ properties.

Definition 1.2. Let $P$ be topological properties such that $P o=(P$ and $\left.T_{0}\right)$ exists. Then a space $(X, T)$ is weakly $P_{0}$ iff its $T_{0}$-identification space $\left(X_{0}, Q(X, T)\right)$ has property $P$. A topological property $P o$ for which weakly $P o$ exists is called a weakly $P o$ property [1]. In the work below, for a topological property $Q,\left(Q\right.$ and $\left.T_{0}\right)$ will be denoted by Qo. In the 1936 paper [12], it was shown that for each space, its $T_{0}$-identification space has property $T_{0}$. Thus, for a topological property $P$ for which $P o$ exists, a space is weakly $P o$ iff its $T_{0}$-identification space has property $P$.

Within the paper [1], it was shown that for a weakly Po property $Q o$, a space is weakly $Q o$ iff its $T_{0}$-identification space is weakly $Q o$, which led to the introduction and investigation of $T_{0}$-identification $P$ properties $[2]$.

Definition 1.3. Let $S$ be a topological property. Then $S$ is a $T_{0}$-identification $P$ property iff both a space and its $T_{0}$-identification space simultaneously shares property $S$. 
In the introductory weakly Po paper [1], it was shown that weakly $P o$ is neither $T_{0}$ nor "not- $T_{0}$ ", where "not- $T_{0}$ " is the negation of $T_{0}$. The need and use of "not- $T_{0}$ " revealed "not- $T_{0}$ " as a useful topological property and tool, motivating the inclusion of the long-neglected properties "not- $P$ ", where $P$ is a topological property for which "not- $P$ " exists, as important properties for investigation and use in the study of topology. As a result, within a short time period, many new, important, fundamental, foundational, never before imagined properties have been discovered, expanding and changing the study of topology.

In past studies of weakly Po spaces and properties, for a classical topological property $Q o$, a special topological property $W$ was sought such that for a space with property $W$, its $T_{0}$-identification space has property Qo, which then implies the initial space has property $W$. If past practices continue, the study of weakly $P o$ spaces and properties would continue to be tedious and never ending. Thus, the question of whether there is a shortcut for the weakly $P_{0}$ space and property search process arose, which was resolved in a recent paper [3].

Answer 1.1. Let $Q$ be a topological property for which both $Q o$ and ( $Q$ and "not- $T_{0}$ ") exist. Then $Q$ is a $T_{0}$-identification $P$ property that is weakly $P o$ and $Q=$ weakly $Q o=\left(Q o\right.$ or $\left(Q\right.$ and "not- $T_{0}$ ") [3].

Answer 1.2. $\left\{Q \mid Q\right.$ is a $T_{0}$-identification $P$ property $\}=\{Q o \mid Q o$ is a weakly $P o$ property $\}=\{Q o \mid Q$ is a topological property and Qo exists $\}$ [3].

Answer 1.3. $\left\{Q \mid Q\right.$ is a $T_{0}$-identification $P$ property $\}=\{Q \mid Q$ is weakly $\left.P_{0}\right\}=\{Q \mid Q$ is a topological property and both $Q o$ and $(Q$ and "not- $T_{0}$ ") exist $\}[3]$.

Thus, major progress was achieved in the study of weakly Po and related properties. If $Q$ is a topological property for which both $Q o$ and 
( $Q$ and "not- $T_{0}$ ") exist, Answer 1.1 quickly and easily gives the shortcut. If $Q$ is a topological property for which $Q=Q o$, then $Q=Q o$ is a weakly Po property, but $Q=Q o$ is not a $T_{0}$-identification $P$ property or weakly Po. Within the recent paper [3], a topological property $W$ that can be both $T_{0}$ and "not- $T_{0}$ " was given that is a $T_{0}$-identification $P$ property that is weakly $P o$ such that $W=$ weakly $Q o$, again making the search process certain, and quick and easy.

Definition 1.4. Let $Q$ be a topological property for which $Q o$ exists. A space $(X, T)$ has property $Q N O$ iff $(X, T)$ is "not- $T_{0}$ " and $\left(X_{0}, Q(X, T)\right)$ has property $Q o[3]$.

In the recent paper [3], it was shown that $Q N O$ exists and is a topological property, and $W=(Q o$ or $Q N O)$ is a $T_{0}$-identification $P$ property that is weakly $P o$ with $W=$ weakly $Q o$. Thus, as stated above, for a topological property $Q$ for which $Q o$ exists and $Q=Q o$, there is a certain, quick, and easy answer.

In the weakly $P_{0}$ paper [4], the use of $T_{0}$ and "not- $T_{0}$ " revealed that $L=\left(T_{0}\right.$ or "not- $T_{0}$ ") is the least of all topological properties. Since the existence of the least topological property $L$ had not even been considered in past studies, its existence required a change in the definition of subspace properties with the removal of $L$ as a subspace property [5]. Prior to the study of weakly $P o$ spaces and properties, it was unknown whether for subspace properties $P$ and $Q$, if ( $P$ and $Q)$ exists. Within the 2016 paper [5], it was shown that for subspace properties $P$ and $Q,(P$ and $Q)$ exists and, thus, is a subspace property. In the paper [5], it was shown that $\mathcal{P}=\{P \mid P$ is a subspace property $\}$ has no least element and in the paper [6], it was shown that $\mathcal{P}$ has strongest element the singleton set property. Since, for the most part, "not- $P$ ", where $P$ is a topological 
property for which "not- $P$ " exists, had been ignored in the study of topology, the introduction and investigation of "not-(subspace property $P$ )" in the paper [4] revealed that for a subspace property $P$, "not(subspace property $P$ )" is not a subspace property, which instantaneously gave many previously unknown examples of topological properties that are not subspace properties. Also, in the paper [4], it was shown that for subspace properties $P$ and $Q$ for which ( $P$ and "not- $Q$ ") exist, ( $P$ and "not- $Q$ ") is not a subspace property, giving many additional new topological properties that are now known to be not subspace properties. Within the 2017 paper [7], similar results were obtained for product properties.

Thus, the study of weakly $P o$ and related properties has exposed a previously unknown, very fruitful territory within topology with properties and tools needed to successfully address previously unasked and/or unanswered questions. Below, the exploration of the recently discovered new territory within topology continues.

\section{Additional Properties of Weakly Po and Related Properties}

Within the paper [8], it was shown that $L$ is weakly $P o$ and $L o=T_{0}$ is a weakly $P o$ property. In the paper [9], it was shown that for a "not- $T_{0}$ " space $(X, T)$, there exists a proper subspace $\left(X T O, T_{X T O}\right)$ that is homeomorphic to $\left(X_{0}, Q(X, T)\right)$.

Definition 2.1. Let $(X, T)$ be a "not- $T_{0}$ " space and let $C_{x}$ be the $T_{0}$-identification space equivalence class containing $x$. Then $X T O$ is a subset of $X$ that contains exactly one element from each equivalence class $C_{x}[9]$. 
Theorem 2.1. Let $(X, T)$ be "not- $T_{0}$ " and let XTO be as defined above. Then $\left(X T O, T_{X T O}\right)$ is homeomorphic to $\left(X_{0}, Q(X, T)\right)[9]$.

Below, the results above are used to further investigate weakly Po and related properties.

Theorem 2.2. Let $(X, T)$ be a "not- $T_{0}$ " space and let $X T O$ be as defined above. Then $\left(X T O, T_{X T O}\right)$ is $T_{0}$.

Proof. Since $\left(X_{0}, Q(X, T)\right)$ is $T_{0}$ and $T_{0}$ is a topological property, then, by the results above, $\left(X T O, T_{X T O}\right)$ is $T_{0}$.

Theorem 2.3. For each space $(X, T)$ that is "not- $T_{0}$ ", $\left(X T O, T_{X T O}\right)$ is a proper, dense subspace of $(X, T)$ that is $T_{0}$.

Proof. By the results above, $X T O$ is a proper subset of $X$ and (XTO, $\left.T_{X T O}\right)$ is $T_{0}$. Let $x \in X \backslash X T O$. Let $O \in T$ such that $x \in O$. Let $y \in X T O$ such that $y \in C_{x}$. Since $C l(\{x\})=C l(\{y\})$, then $y \in O$. Hence, $X T O$ is dense in $(X, T)$.

Theorem 2.4. Let $Q$ be a topological property for which ( $Q$ and "not- $T_{0}$ ") exists. Then the following are equivalent: (a) $Q$ is a $T_{0}$-identification P property, (b) $Q$ is a weakly Po property, (c) for each space $(X, T)$ with property ( $Q$ and "not- $T_{0}$ ”), (XTO, $\left.T_{X T O}\right)$ has property $Q$, and (d) for each space $(X, T)$ with property ( $Q$ and "not- $T_{0}$ "), (XTO, $\left.T_{X T O}\right)$ has property Qo.

Proof. By the results above, (a) and (b) are equivalent.

(b) implies (c): Let $(X, T)$ be a space with property ( $Q$ and "not- $T_{0}$ "). Since $Q$ is weakly $Q$, then $\left(X_{0}, Q(X, T)\right)$ has property $Q$. Since $(X, T)$ has property "not- $T_{0}$ ", then $\left(X T O, T_{X T O}\right)$ is 
homeomorphic to $\left(X_{0}, Q(X, T)\right)$ and since $Q$ is a topological property, $\left(X T O, T_{X T O}\right)$ has property $Q$.

By Theorem 2.2, (c) implies (d).

(d) implies (b): Let $(X, T)$ be a space with property ( $Q$ and not- $T_{0}$ ”).

Since $\left(X T O, T_{X T O}\right)$ has property $Q o$, then both $Q o$ and ( $Q$ and not- $T_{0}$ ”) exist and, by Answer 1.3 above, $Q$ is weakly $P$.

In the 2015 paper [2], it was proven that for topological properties $Q$ and $W$, which are both weakly $P o$, weakly $Q o=$ weakly $W_{o}$ iff $Q o=W o$. Could $Q=W$ be added as an equivalent statement?

Theorem 2.5. Let $Q$ be a topological property that is weakly Po. Then $Q$ is a $T_{0}$-identification $P$ property and $Q=$ weakly $Q$.

Proof. By Answer 1.3 above, both $Q o$ and ( $Q$ and "not- $T_{0}$ ") exist. Then by Answer 1.1 above, $Q$ is a $T_{0}$-identification $P$ property and $Q=$ weakly $Q o$.

Theorem 2.6. Let $Q$ and $W$ be topological properties, both of which are weakly Po. Then the following are equivalent: (a) weakly Qo = weakly $W o$, (b) $Q o=W o$, and (c) $Q=W$.

Proof. By the results above, (a) and (b) are equivalent.

(a) implies (c): By Theorem 2.5, $Q=$ weakly $Q o$ and $W=$ weakly $W_{o}$ and, since weakly $Q o=$ weakly $W o$, then $Q=W$.

Clearly, (c) implies (a).

Theorem 2.7. Let $Q$ and $W$ be topological properties, both of which are weakly Po. Then the following are equivalent: (a) weakly Qo implies weakly Wo, (b) Q implies W, and (c) Qo implies Wo.

Proof. (a) implies (b): Since $Q=$ weakly $Q o, W=$ weakly $W o$, and weakly $Q o$ implies weakly $W o$, then $Q$ implies $W$. 
Clearly (b) implies (c).

(c) implies (a): Let $(X, T)$ be weakly $Q o$. Then $\left(X_{0}, Q(X, T)\right)$ is $Q o$, which implies $\left(X_{0}, Q(X, T)\right)$ is $W o$, which implies $(X, T)$ is weakly Wo. Thus, weakly Qo implies weakly Wo.

\section{Weakly Po for Each Existent Qo}

Definition 3.1. A space $(X, T)$ has property $S M$ iff for $x$ and $y$ in $X$ such that $C l(\{x\}) \neq C l(\{y\})$, there exists an open set $U$ containing only one of $x$ and $y$.

Theorem 3.1. Every space has property SM.

Proof. Let $(X, T)$ be a space. Let $x$ and $y$ be elements in $X$ such that $C l(\{x\}) \neq C l(\{y\})$. Then $x \notin C l(\{y\})$ or $y \notin C l(\{x\})$, say $x \notin C l(\{y\})$. Then $x \in U=X \backslash C l(\{y\})$ is open and $y \notin U$. Thus $(X, T)$ has property $S M$.

Corollary 3.1. SM is a topological property.

Theorem 3.2. Let $(X, T)$ be a space. Then $(X, T)$ has property L iff for $x$ and $y$ in $X$ such that $C l(\{x\}) \neq C l(\{y\})$, there exists an open set $U$ containing only one of $x$ and $y$.

Proof. By Theorem 3.1, if $(X, T)$ has property $L$, then $(X, T)$ has property $S M$. Thus $L$ implies $S M$ and, since $L$ is the least of all topological properties, then $L=S M$.

Definition 3.2. Let $(X, T)$ be a space and for each $x \in X$, let $C_{x}$ be the $T_{0}$-identification class containing $x$. Then $O X T O$ is a subset of $X$ containing exactly one element from each equivalence class $C_{x}$.

Note that if $(X, T)$ is "not- $T_{0}$ ", then a subset $O X T O$ of $X$ is an XTO subset of $X$. 
Theorem 3.3. Let $(X, T)$ be a space. Then $\left(\right.$ OXTO, $\left.T_{\text {OXTO }}\right)$ is homeomorphic to $\left(X_{0}, Q(X, T)\right)$.

Proof. By Theorem 3.2, $(X, T)$ is $\left(T_{0}\right.$ or "not- $T_{0}$ "). Consider the case that $(X, T)$ is $T_{0}$. Then $X=O X T O$ and $(X, T)=\left(O X T O, T_{O X T O}\right)$ is $T_{0}$. Since a space $(Y, S)$ is $T_{0}$ iff the natural map $N:(Y, S) \rightarrow$ $\left(Y_{0}, Q(Y, S)\right)$ is a homeomorphism [10], then $\left(\right.$ OXTO, $\left.T_{\text {OXTO }}\right)$ is homeomorphic to $\left(X_{0}, Q(X, T)\right)$. Thus, consider the case that $(X, T)$ is "not- $T_{0}$ ". Then $O X T O=X T O$ and, by Theorem 2.1, $\left(\right.$ OXTO, $\left.T_{O X T O}\right)$ is homeomorphic to $\left(X_{0}, Q(X, T)\right)$.

Definition 3.3. Let $Q$ be a topological property for which $Q o$ exists. Then a space $(X, T)$ has property $W Q$ iff $\left(O X T O, T_{O X T O}\right)$ has property Qo.

Within the paper [3], it was shown that for a topological property $Q$ such that $Q o$ exists, $(Q o$ or $Q N O)$ is a $T_{0}$-identification $P$ property, ( $Q o$ or $Q N O)=$ weakly $(Q o$ or $Q N O) o=$ weakly $Q o$, and $(Q o$ or $Q N O)$ is a weakly $P o$ property, which is used below.

Theorem 3.4. Let $Q$ be a topological property for which Qo exists and let $(X, T)$ be a space. Then $(X, T)$ has property WQ iff $(X, T)$ has property (Qo or $Q N O)$.

Proof. Suppose $(X, T)$ has property $W Q$. Then $\left(\right.$ OXTO, $\left.T_{\text {OXTO }}\right)$ has property $Q o$ and, since $\left(O X T O, T_{O X T O}\right)$ is homeomorphic to $\left(X_{0}, Q\right.$ $(X, T))$, then $\left(X_{0}, Q(X, T)\right)$ has property Qo. Since Qo exists, then (Qo or $Q N O)$ is a $T_{0}$-identification $P$ property and $(Q o$ or $Q N O)=$ weakly $Q o$. Thus, a space $(Y, S)$ is $(Q o$ or $Q N O)$ iff $\left(Y_{0}, Q(Y, S)\right)$ is $Q o$, and, since $\left(X_{0}, Q(X, T)\right)$ is $Q o$, then $(X, T)$ is $(Q o$ or $Q N O)$. 
Conversely, suppose $(X, T)$ has property (Qo or $Q N O)$. Then $\left(X_{0}, Q(X, T)\right)$ has property $Q$ a and, since $\left(\right.$ OXTO, $\left.T_{\text {OXTO }}\right)$ is homeomorphic to $\left(X_{0}, Q(X, T)\right)$, then $\left(O X T O, T_{O X T O}\right)$ has property $Q$. Hence $(X, T)$ has property $W Q$.

Therefore, $W Q=(Q o$ or $Q N O)$.

Since both $Q o$ and $Q N O$ are topological properties, then $W Q$ is a topological property.

Corollary 3.2. Let $Q$ be a topological property for which Qo exists. Then $W Q$ is the special property for which a space has property WQ iff its $T_{0}$-identification space has property Qo.

Below the results above are applied to determine $W\left(T_{1}\right)$.

Let $(X, T)$ have property $W\left(T_{1}\right)$.

Then $\left(\right.$ OXTO, $\left.T_{\text {OXTO }}\right)$ has property $\left(T_{1}\right) o=T_{1}$. Suppose there exists a $x \in X$ such that $C_{x} \neq C l(\{x\})$. Since $C_{x} \subseteq C l(\{x\})$, let $y \in C l(\{x\})$ that is not in $C_{x}$. Let $u, v \in O X T O$ such that $x \in C_{u}$ and $y \in C_{v}$. Then $u$ and $v$ are distinct elements in OXTO and since $\left(O X T O, T_{O X T O}\right)$ is $T_{1}$, there exists and open set $V$ containing $v$ and not $u$. Let $O \in T$ such that $V=O \cap O X T O$. Then $y \in O$ and $x \notin O$, which contradicts $y \in C l(\{x\})$. Thus, for each $x \in X, C_{x}=C l(\{x\})$ and $\{C l(\{x\}) \mid x \in X\}$ is a decomposition of $X$.

Conversely, suppose $\{C l(\{x\}) \mid x \in X\}$ is a decomposition of $X$. Let $u$ and $v$ be distinct elements of OXTO. Then $\{v\}=C l(\{v\}) \cap O X T O$ is closed in OXTO. Hence singleton sets are closed in OXTO and (OXTO, $\left.T_{O X T O}\right)$ is $T_{1}$. 
Hence, a space $(X, T)$ has property $W\left(T_{1}\right)$ iff $\{C l(\{x\}) \mid x \in X\}$ is a decomposition of $X$.

Past work in $T_{0}$-identification spaces and weakly $P$ o spaces verify the result above. The application above is not required, but it does add comfort to use of the work above.

In the paper [3], it was shown that if $Q$ is a topological property for which both $Q o$ and ( $Q$ and not- $T_{0}$ ”) exist, then $Q$ is a $T_{0}$-identification $P$ property, $Q N O=\left(Q\right.$ or "not- $T_{0}$ ” $)$, and $Q=$ weakly $Q o=(Q o$ or $Q N O)=$ ( $Q$ o or $\left(Q\right.$ and "not- $T_{0}$ ”), which greatly simplifies the above process.

Corollary 3.3. Let $Q$ be a topological property for which both Qo and $\left(Q\right.$ and "not $-T_{0}$ ") exist. Then $W Q=Q$.

\section{References}

[1] C. Dorsett, Weakly $P$ properties, Fundamental Journal of Mathematics and Mathematical Sciences 3(1) (2015), 83-90.

[2] C. Dorsett, $T_{0}$-Identification $P$ and weakly $P$ properties, Pioneer Journal of Mathematics and Mathematical Sciences 15(1) (2015), 1-8.

[3] C. Dorsett, Complete characterizations of weakly $P o$ and related spaces and Properties, Journal of Mathematical Sciences: Advances and Applications 45, 97-109.

[4] C. Dorsett, Weakly $P$ corrections and new fundamental topological properties and facts, Fundamental Journal of Mathematics and Mathematical Sciences 5(1) (2016), 11-20.

[5] C. Dorsett, New properties tools, and changes for subspace properties and singleton set spaces, Pioneer Journal of Mathematics and Mathematical Sciences 17(2) (2016), 78-85.

[6] C. Dorsett, Intersection existence and least and strongest properties for topological properties product properties subspace properties and properties that are weakly $P o$, Pioneer Journal of Mathematics and Mathematical Sciences 19(1), 37-44.

[7] C. Dorsett, Pluses and needed changes in topology resulting from additional properties, Far East Journal of Mathematical Sciences 101(4) (2017), 803-811. 
[8] C. Dorsett, Corrections and more insights for weakly $P o, T_{0}$-identification $P$, and their negations, Fundamental Journal of Mathematics and Mathematical Sciences $8(1), 1-7$.

[9] C. Dorsett, Additional properties for weakly Po and related properties, and subspace properties, accepted by Pioneer Journal of Mathematics and Mathematical Sciences.

[10] C. Dorsett, New characterizations of separation axioms, Bulletin of the Calcutta Mathematical Society 99(1) (2007), 37-44.

[11] W. Dunham, Weakly Hausdorff spaces, Kyungpook Math. J. 15(1) (1975), 41-50.

[12] M. Stone, Applications of Boolean algebras to topology, Mat. Sb. 1 (1936), 765-771. 